\title{
THE EARLY DETECTION OF THE EMERALD ASH BORER (EAB) USING MULTI- SOURCE REMOTELY SENSED DATA
}

\author{
B. $\mathrm{Hu}^{1 *}$, F. Naveed ${ }^{1}$, F. Tasneem ${ }^{1}$, C. Xing ${ }^{1}$ \\ ${ }^{1}$ Dept. of Earth and Space Science and Engineering Department, York University, 4700 Keele Street, Toronto, Canada \\ - baoxin@yorku.ca/ fazanham@my.yorku.ca/farah93@yorku.ca/xingchen@yorku.ca
}

Commission III, WG III/4

KEY WORDS: Tree health, Hyperspectral, Species identification, LiDAR, Chlorophyll content, high spatial resolution imagery

\begin{abstract}
:
The objectives of this study were to exploit the synergy of hyperspectral imagery, Light Detection And Ranging (LiDAR) and high spatial resolution data and their synergy in the early detection of the EAB (Emerald Ash Borer) presence in trees within urban areas and to develop a framework to combine information extracted from multiple data sources. To achieve these, an object-oriented framework was developed to combine information derived from available data sets to characterize ash trees. Within this framework, an advanced individual tree delineation method was developed to delineate individual trees using the combined high-spatial resolution worldview-3 imagery was used together with LiDAR data. Individual trees were then classified to ash and non-ash trees using spectral and spatial information. In order to characterize the health state of individual ash trees, leaves from ash trees with various health states were sampled and measured using a field spectrometer. Based on the field measurements, the best indices that sensitive to leaf chlorophyll content were selected. The developed framework and methods were tested using worldview-3, airborne LiDAR data over the Keele campus of York University Toronto Canada. Satisfactory results in terms of individual tree crown delineation, ash tree identification and characterization of the health state of individual ash trees. Quantitative evaluations is being carried out.
\end{abstract}

\section{INTRODUCTION}

Emerald Ash Borer (EAB, Agrilus planipennis Fairmaire) is an invasive insect species that attacks all species of ash trees (Fraxinus spp). It was first found in North America in 2002 and has become established in several states in the United States of America and in Ontario, Canada. The beetle has caused the death of millions of ash trees during the past decade. Despite substantial research and control efforts, the beetle has continued to spread to new areas. The ash trees attacked by EABs typically die within 3 to 5 years and often show serious decline within 2 years. Due to the aggressive nature of EAB infestation, the earliest possible detection of the infestation is crucial to help reduce the spread and damage of EAB. It would be ideal to be able to detect the beetle when its density is still low and before signs and symptoms are obvious. Commonly used methods include sticky traps baited with an attractant and branch sampled gallery counts (Ryall et al. 2011). Even though these field survey methods have been proved effective, they are labour-intensive Alternative solutions have sought to exploit the increasingly available remote sensing technologies.

For the past decade, studies have been conducted to assess the capabilities of various remote sensing technologies to predict ash tree decline caused by EAB infestation. Souci et al. (2009) reported an EAB detection project in the city of Milwaukee, USA using hyperspectral SpecTIR imagery (with a spatial resolution of $1 \mathrm{~m}$ by $1 \mathrm{~m}$ ) and LiDAR (Light Detection And Ranging) data. In their project, ash trees were classified from hyperspectral imagery using training samples and the results were improved by information derived from LiDAR data using geographic information system (GIS) tools. The classification was deemed to be successful, even though quantitative assessment was not reported. They also concluded that it was critically important to collect numerous field spectra from diverse trees in terms of spatial location, species, state, and understory composition. In a previous study of ours (Zhang et al 2014) improved the prediction of $\mathrm{EAB}$ infestation by combining spectral and spatial information derived from the hyperspectral SpecTIR imagery and high spatial resolution Google Earth imagery, respectively. An object oriented method was employed with individual tree crowns as the basic units. However, the individual ash trees were delineated manually. In this study, we establish a framework to identify ash trees automatically and effectively and characterize their EAB infestation stages within urban environments using field spectrometer data, hyperspectral imagery, together with very high spatial resolution imagery and LiDAR data. To integrate effectively information from different sources, we adopted an object-oriented approach and employed individual tree crowns as the basic unit. For the first time, a complete work flow from individual tree crown delineation to ash tree identification to the characterization of EAB infestation was proposed. The proposed framework and methods were tested using data over the Keele campus of York University, Toronto Ontario.

\section{STUDY AREA AND DATA USED}

The study area is located in the Keele campus of York University, Toronto, Canada $\left(43.7735^{\circ} \mathrm{N}, 79.5019^{\circ} \mathrm{W}\right)$. There are trees of various species along roads and in woodlots. Between 2014 and 2016, 1700 trees were sampled with their species identified and, their heights, crown sizes and DBH measured by CSBO (Campus Services and Business Operations). Among them, 130 are ash trees. These trees were used to validate the methods developed for individual tree delineations and identification. Several remotely sensed data over the campus are available for this study. They include the Worldview-2 imagery obtained on July 25, 2012 and July 21, 2016, Worldview-3 imagery on August 31, 2017, and airborne LiDAR data acquired in April 2015 with a point density of 10 points per square meters (Airborne Imaging, 2015). The LiDAR data was collected using a Leica ALS 70 LiDAR instrument at a flying height of 1300 meters with a Pulse Rate Frequency (PRF) of $400 \mathrm{kHz}$. The horizontal accuracy of the collected LiDAR data was $30 \mathrm{~cm}$ and a vertical accuracy (on flat hard surfaces) of $10 \mathrm{~cm}$. A digital elevation model (DEM) and digital surface model (DSM) with the same spatial resolution 
as the Worldview-3 imagery were subsequently generated from the LiDAR data cloud. The CHM was derived as the difference between the DSM and DEM, which was then smoothed with a 3 by 3 Gaussian low-pass filter to eliminate noise. The true colour composite of the Worldview-3 imagery and the LiDAR DSM model are shown in Figure 1 and Figure 2, respectively. To exploit the use of hyperspectral imagery on the retrieval of leaf chlorophyll content and in turn to characterize the health state of individual ash trees, leaf samples were collected from 10 ash trees and leaf reflectance and transmittance were measured using Ocean Optics field spectrometer.

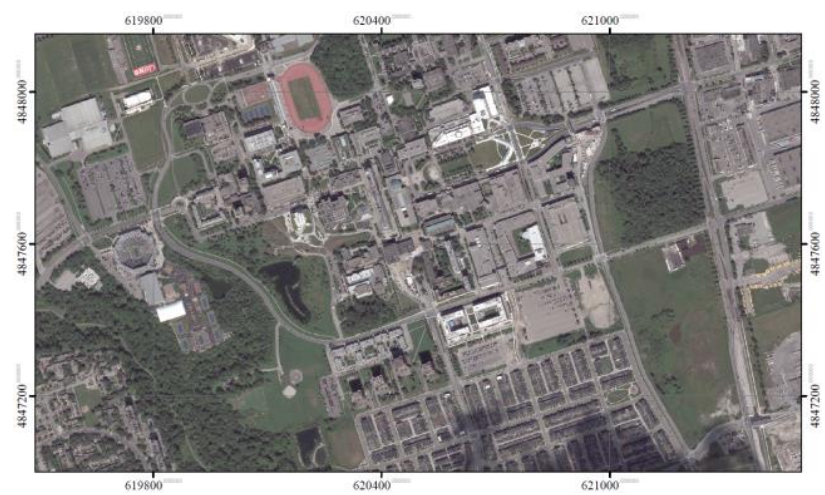

Figure 1: The true colour composite of the Worldview-3 imagery of the study area.

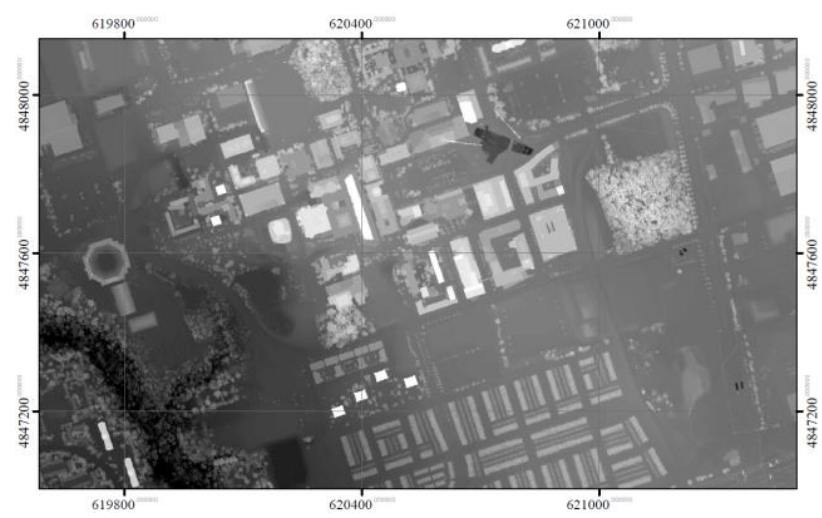

Figure 2: The digital surface model (DSM) derived from the airborne LiDAR data over the study area. The lowest elevation (dark) is 170.80 meters, while the highest one (bright) is 255.88 meters.

\section{METHOD}

The basic idea underlying the developed methods was as follows: individual trees were first extracted from the high spatial resolution worldview-3 imagery and treated as basic units for subsequent analyses. These trees were then classified into different species using spectral, textural, and structural information derived from the worldview data. For each ash tree, its state of health and EAB infestation stage were determined using data from imaging spectrometer and worldview-3.

\subsection{Individual tree crown delineation}

In this study individual tree crown delineation was performed using both Airborne LiDAR data and Worldview 3 imagery. The vertical profiles of the trees from the LiDAR data were initially used to detect 'tree tops' in the study area, using a multiscale approach (Hu et al., 2014). This multiscale approach detected the largest horizontal cross sections of individual trees, as compared with a single tree top point is most of individual tree delineation methods. This approach also minimized the false tree top detection originating from complex deciduous tree structure.

With the seeds identified, segmentation was performed using a modified seeded region growing algorithm based on neutrosophic logic (Shan et al., 2008) using the Worldview-3 imagery. Traditional methods of region growing rely on a local measure of distance to merge pixels to their respective seeds, whereas in neutrosophic based segmentation the criterion for seed growth is analysed using contextual information. In the neutrosophic domain, the criterion for the seed growth depends on two quantities: degree of truth and level of indeterminacy. The degree of truth is a measure of the normalized difference between the pixel value, of the pixel to be merged to the seed, and the mean of the seed. Degree of indeterminacy introduces a measure of variance of the small circular neighbourhood surrounding the pixel. In this study, a small degree of indeterminacy implied that the region around the pixel was homogeneous hence representing a single tree type. On the contrary a large indeterminacy value was indicative of a more heterogeneous region hence indicating presence of another tree crown type nearby. For merging the pixels to their respective seeds, a rule based approach was used. If the degree of indeterminacy was smaller than a threshold value than the degree of truth of the individual pixel was calculated, as it was indicative of a less noisy region around the pixel. A higher degree of truth consequently implied that the pixel was similar in structural and spectral domain to the seed. A level of indeterminacy higher than the threshold value was indicative of the presence of another crown type and hence the degree of truth of a small circular region surrounding the pixel was calculated instead. A sigmoid function was used determine the threshold degree of truth, given the level of indeterminacy. A higher indeterminacy value implied that the pixel was in a nosier region and individual pixel values were not reliable enough to make a merge decision. The mathematical notation of the criteria is illustrated in Equation (1) (Shan et al., 2008).

$$
\begin{aligned}
& \forall P_{i} \in J:\left\{\left(( I ( P _ { i } ) < I _ { T h r } \wedge T ( P _ { i } ) > T _ { T h r } ) \vee \left(I\left(P_{i}\right)>I_{T h r} \wedge\right.\right.\right. \\
& \left.\left.\left.T^{\prime}\left(P_{i}\right)>T^{\prime} T h r\right)\right) \rightarrow P_{i} \in S_{j}\right\}
\end{aligned}
$$

where $P_{i}=$ A given pixel in the image domain $J$

$$
\begin{aligned}
& S_{j}=\text { A given segment } \\
& I\left(P_{i}\right)=\text { The indeterminacy value around pixel } P_{i} \\
& T\left(P_{i}\right)=\text { The degree of truth of pixel } P_{i} \\
& T^{\prime}\left(P_{i}\right)=\text { The degree of truth of the neighbourhood of } P_{i} \\
& T^{\prime}{ }_{T h r}=F\left(I\left(P_{i}\right)\right)=\text { The sigmoid function }
\end{aligned}
$$

\subsection{The identification of individual ash trees}

Most of the existing classification methods for species identification are targeted for a number of species types. However, the interest often lies in one or a few of the species types studied. In such circumstances, the "standard" classification methods that aim to optimize the overall accuracy of all classes may not be appropriate for the specific class/classes of interest in terms of the features and determinant decision rules used. Foody et al. (2005) demonstrated that by specifically minimizing the omission and commission errors of classifying the species of interest, sycamore in this case, rather than the general mis-classification errors for all species types in the scene, the classification accuracy for the species was increased from $46.6 \%$ and $64.6 \%$ to $82.3 \%$ and $93.3 \%$ for the producer's accuracy and user's accuracy, respectively. In the study of Foody et al (2005), the features used for classifications were the same. In our opinion, if the features had been selected specifically to maximize the separation between sycamore and other species, the 
results would have been better. To the best of our knowledge, the existing "standard" methods cannot generate satisfactory classification results for ash trees and no methods have been designed specifically for ash tree identification.

To design a classification scheme for ash tree identification, we compiled known facts on ash trees and exploited the feasibility of employing them to identify ash trees from available remotely sensed data. Specifically, we derived the features sensitive to leaf chlorophyll content with the hypothesis that trees of different species tend to have different composition of pigments. Since the preferred hyperspectral imagery is not available, the Worldview3 imagery was used and it is expected the spectral band along the red-edge in the Worldview-3 imagery would provide sufficient information on leaf pigment content together with other traditional multispectral channels. With the high-spatial resolution WV-3 panchromatic band, we also derived textural information of individual crowns that may reflect the characteristics of the crown shape, branch structure, crown size, foliage type and orientation. The ash tree is quite unique species among the deciduous species on the campus. Ash is the only species in the study area that exhibits opposite branching and has compound leaf. Both the statistical-based GLCM (Grey Level Co-occurrence Matrix) measures and structural-based LBP (local binary pattern) indices were calculated and employed for classification ( $\mathrm{Li}$ et al., 2015). In addition, the structural information reflecting the shape of individual trees were derived from the LiDAR data ( $\mathrm{Li}$ et al., 2013). All of the features were used to separate ash trees from other species. The support vector machine (SVM) method was employed in this study.

\subsection{Characterization of EAB infestation}

In order to select the best indices to characterize the infestation stage of individual ash trees, we collected leaves of 8 ash trees and measured the reflectance and transmittance of 5 leaves for each sampled tree using the ocean optics spectrometer. Among the 8 trees, 5 are healthy and 3 are not. Figure 3 shows two of the sampled trees. The measured reflectance and transmittance were processed by using the Ensemble Empirical Mode Decomposition (EEMD) (Huang et al., 1995) to remove noise. This method was selected due to its capability of removing noise while preserving features. Figure 4 shows the original reflectance measurement and the noise reduced reflectance resulted from the EEMD method. The result generated using the traditional low-pass filter noise reduction method is shown in Figure 4 as comparison. The healthy trees tend to have higher chlorophyll content than the unhealthy ones, which leads to the differences in the reflectance between them. We derived the leaf chlorophyll content using physical model inversion method based on the PROSPECT model (Jacquemoud and Baret, 1990) and tested a number of indices that are sensitive to leaf chlorophyll content. They were chlorophyll vegetation index (CVI) (Vincini et al., 2008), CI-G and CI-Re (Gitelson et al., 2003), and the triangular greenness index (TGI) (Raymond et al., 2013). $t$ test was carried out to determine if there was a significant difference in the leaf chlorophyll content and in the calculated vegetation indices between healthy and unhealthy trees. The top indices were applied to Worldview-2 imagery. For each individual ash tree, the indices were calculated using Worldview-2 data acquired in 2012 and 2016, respectively. The results obtained in 2016 were used to validate those from 2012. The rationale behind this is that if an ash tree was detected as infested by $\mathrm{EAB}$ at an early stage based on its pigment content in 2012, the situation would be worse in 2016 with this tree either declined significantly or died. Based on the indices selected, unsupervised classification was carried out to separate the ash trees into two categories: healthy and unhealthy. For this investigation, only the sunlit pixels within an ash tree segment were used.
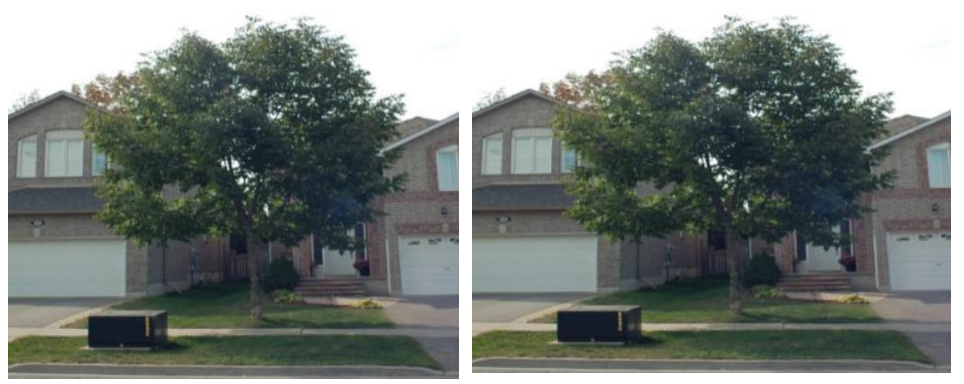

Figure 3. Two of the sampled ash trees.

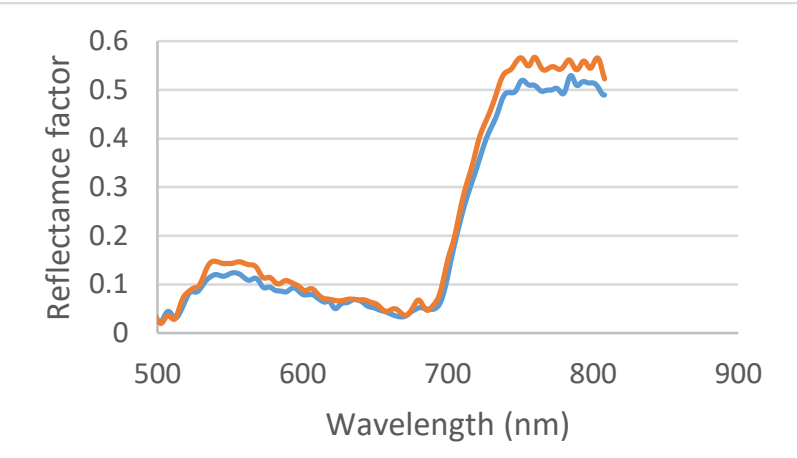

Figure 4. The reflectance spectrum of a healthy leaf (blue) and an unhealthy leaf (orange).

\section{RESULTS AND DISCUSSION}

Even though the individual tree crowns in the study area were delineated using the proposed algorithm, the results for two subset areas are shown in Figures 5-8 to achieve a better visualization. One area is a woodlot and the other with trees along streets. Based on visual inspection, the segments generated by the proposed method provide satisfactory results. However, there are some problems occurring in the woodlot area where three crowns tend to overlap with each other. Some of segments cover more than the tree crowns, and for some segments, the shapes tend to be elongated, far away from the circular shape of tree crowns. These issues would be tackled in future work.

In term of the ash tree identification, preliminary results show that the addition of the red-edge channel in the Worldview imagery significantly increases the classification accuracy, if only spectral information was used. Combining structural information derived from LiDAR data with the spectral information from Worldview-3 imagery improved ash tree identification. Detailed numerical analysis is being carried out.

Regarding the characterization of the health stage of ash trees, the leaf chlorophyll content, the four vegetation indices calculated for the leaves from 8 sampled ash trees are shown in Table 1. It is clear that the leaf chlorophyll content, the CVI, CI-G and CIRe were significantly different between healthy and unhealthy leaves. The difference was not clearly considering the high standard deviation with the unhealthy leaves. t-test results confirmed the observations at the significant level of 0.05 . As a 
result, the three indices CVI, CI-G and CI-Re were employed to characterize the health state of ash trees using World-view imagery.

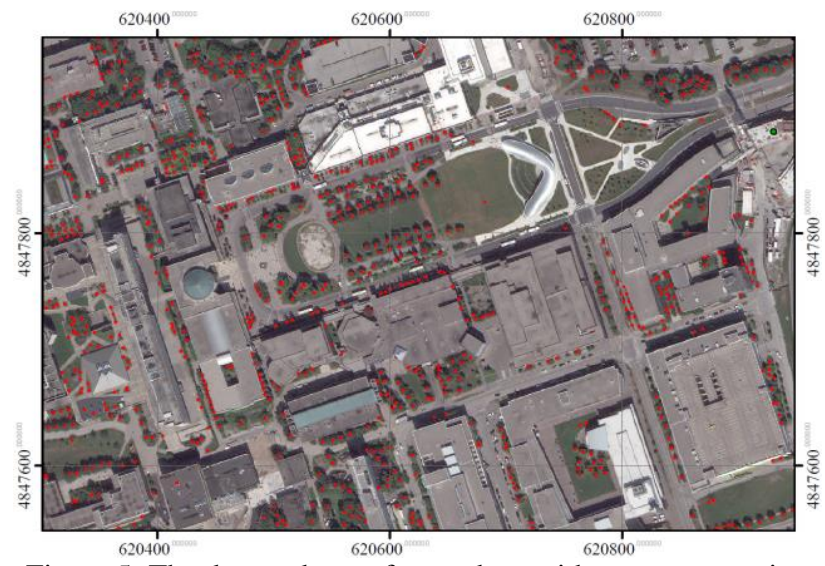

Figure 5: The detected trees for a subset with street trees using the combined worldview-3 imagery and airborne LiDAR data. The segments are overlaid on the true colour composite of workview-3 imagery.

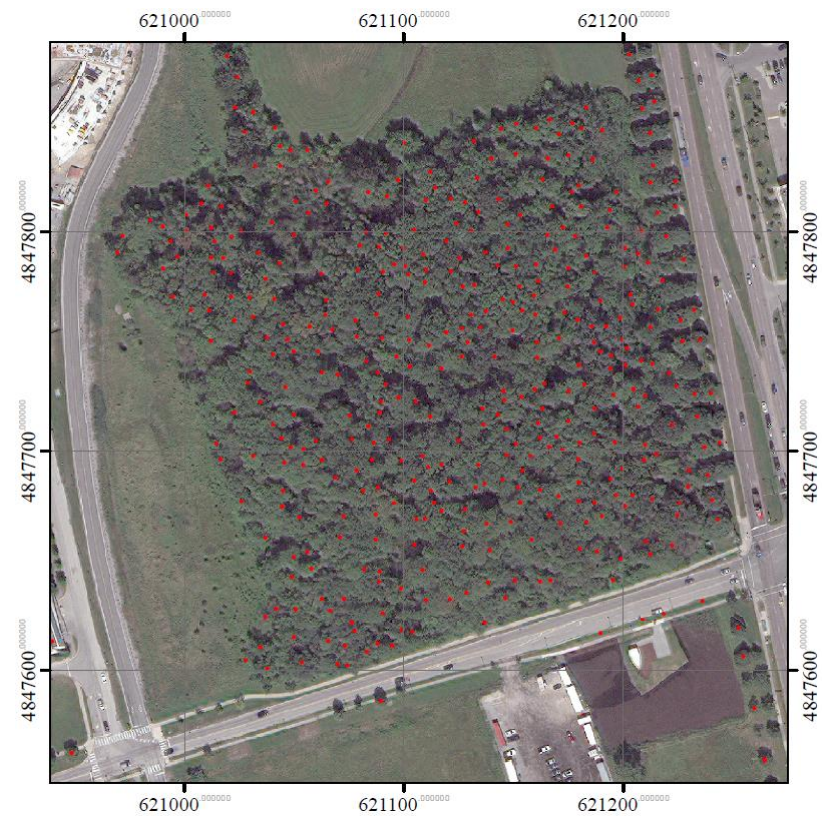

Figure 6: The detected trees for a woodlot subset using the combined worldview- 3 imagery and airborne LiDAR data. The segments are overlaid on the true colour composite of workview-3 imagery.

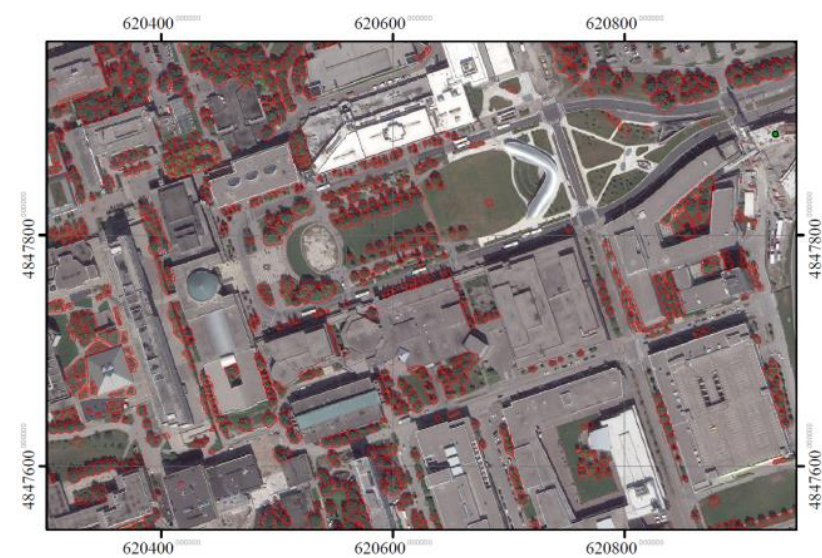

Figure 7: The tree segments for the subset with street trees.

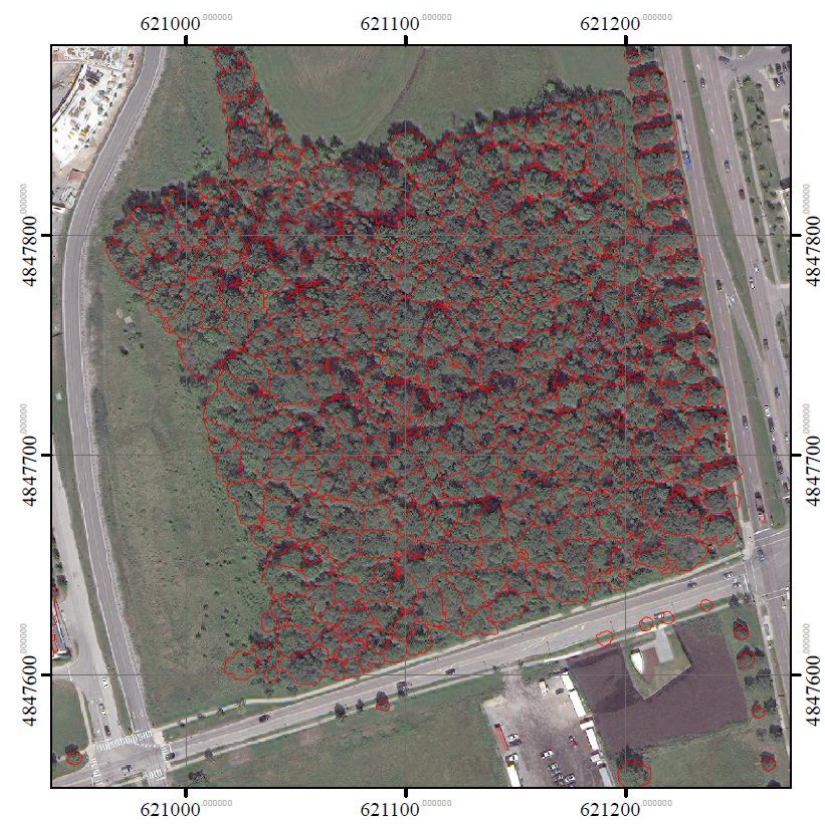

Figure 8: The tree segments for the woodlot subset.

\begin{tabular}{|l|l|l|l|l|}
\hline \multirow{2}{*}{} & \multicolumn{2}{|l|}{ Healthy } & \multicolumn{2}{l|}{ Unhealthy } \\
\cline { 2 - 5 } & Mean & Std & Mean & Std \\
\hline $\begin{array}{l}\text { Chlorophyll } \\
\text { content }\end{array}$ & 55.07 & 3.32 & 41.12 & 4.28 \\
\hline CI-Re & 3.48 & 0.50 & 2.22 & 0.40 \\
\hline CI-G & 3.75 & 0.28 & 1.78 & 0.28 \\
\hline CVI & 2.27 & 0.33 & 1.78 & 0.80 \\
\hline TGI & 6.95 & 1.0 & 9.55 & 3.6 \\
\hline
\end{tabular}

Table 1: The mean and standard deviation (Std) calculated for the leaves samples collected from 5 healthy ash trees and 3 unhealthy trees. 


\section{CONCLUSIONS}

The framework developed to segment individual tree crown, identify ash trees and characterize their $\mathrm{EAB}$ infestation was demonstrated to be promising based on the data obtained from Worldview and airborne LiDAR data over the Keele campus, York University, Toronto Canada. Most of the evaluations were based on visual inspection. Vigorous quantitative analysis are needed and is being pursued. With the framework, objectoriented methods were developed to integrate effectively information from different remote sensing data. The fusion of spectral and height information derived from high spatial resolution satellite imagery and LiDAR data proved to be necessary and effective in the delineation of individual tree crowns. With our ITC delineation method, satisfactory results were obtained.

In terms of the identification of ash trees, spectral, spatial, and structural features were used. These features were calculated for each tree segments from wordview-3 imagery and LiDAR data, respectively. Both random forest and SVM methods were used in this study. Feature selection was integrated in the classification process. Detailed analysis of the significance of these features is currently under investigation. Tests conducted with trees in the study site demonstrated satisfactory results. Caution should be taken in that the classification tests were based on a few training and testing samples, and further research with more field samples is needed.

The use of hyperspectral data to quantify the health of ash trees achieved expected results. Unhealthy ash trees exhibited lower reflectance at the red edge and NIR bands than healthy ash trees. The vegetation indices used in this study proved to be very useful in the separation between healthy and unhealthy ash trees. Further validation is required.

\section{ACKNOWLEDGEMENTS}

The authors would like to thank York University library and CSBO for the LiDAR and tree inventory data, respectively. The authors are also grateful for Drs. B. Hall and J. Wang for useful discussions, Andy Jung for his help with the tree locations, and Paul Hambidge and Leighanne Howard if the city of Peterborough for their help in leave sampling. We would like to acknowledge the financial support provided by the Natural Sciences and Engineering Research Council (NSERC) of Canada and Esri Canada.

\section{REFERENCES}

Airborne Imaging, 2015. Greater Toronto Area LIDAR Imaging. Accessed via York University library, Toronto, Ontario, Canada.

Foody, G. M., Atkinson, P. M., Gething, P. W., et al., 2005. Identification of specific tree species in ancient seminatual woodland from digital aerial sensor imagery, Ecological Applications, 15(4), pp. 1233-1244.

Gitelson, A. A., Yuri, G. Y., and Merzlyak, M. N., 2003. Relationships between leaf chlorophyll content and spectral reflectance algorithms for non-destructive chlorophyll assessment in higher plants, Journal of Plant Physiology 160, pp. 271-282.
Hu, B., Li, J., Jing, L. and Judah, A., 2014. Improving the efficiency and accuracy of individual tree crown delineation from high-density LiDAR data, International Journal of Applied Earth Observation and Geoinformation, 26, pp. 144155.

Huang, N. E., Shen, Z., Long, S. R., Wu, M. C., Shih, E. H., Q. Zheng, Q., Tung, C. C., and Liu, H. H., 1998. The empirical mode decomposition method and the Hilbert spectrum for non-stationary time series analysis, Proc. Roy. Soc. London, 454A, pp. 903-995.

Jacquemoud, S. and Baret, F., 1990. PROSPECT: A Model of Leaf Optical Properties Spectra. Remote sensing of Environment, 34, pp.75-91.

Li. J., Hu, B., and Woods, M., 2015. A two-level approach for identification of species importance to the winter habitat of white-tailed deer among coniferous trees in central Ontario forest. International Journal of Applied Earth Observation and Geoinformation, 8(4), pp.1487-1497.

Li, J, Hu, B. and Noland, T., 2013. Classification of tree species based on structural features derived from high density LiDAR data. Agricultural and Forest Meteorology 171- 172, pp.104114.

Ryall, K. L., Fidgen, J. G., and Turgeon, J. J., 2011. Detectability of the emerald urban tree by using branch Samples, Environmental Entomology, 3(40), pp.679-688.

Raymond, E., Doraiswamy, P. C., McMurtrey, J. E. et al., 2013. A visible band index for remote sensing leaf chlorophyll content at the canopy scale, International Journal of Applied Earth Observation and Geoinformation 21, pp. 103-112.

Shan, J., Cheng, H. D., Wang, Y., 2008. A completely automatic segmentation method for breast ultrasound images using region growing. In Proceedings of the 9th Int. Conference on Computer Vision, Pattern Recognition, \& Image Processing, pp.1-4.

Souci, J. S., Hanou, I, and Puchalski, D., 2009. High resolution remote sensing analysis for early detection and response planning for emerald ash borer, Photogrammetric Engineering and Remote Sensing, 75(8), pp. 905-909.

Vincini, M., Frazz, E., and D'Alessio, P., 2008, A broad-band leaf chlorophyll vegetation index at the canopy scale, Precision Agriculture, 9(5), pp. 303-319.

Zhang, K., Hu, B., and Robinson, J. 2014. Early detection of Emerald Ash Borer (EAB) infestation using multi-sourced data: a case study in the town of Oakville, Ontario, Canada. J. of Applied Remote Sensing, 8(1), 083602. doi:10.1117/1.JRS.8.083602 